\title{
From prevention to management of the complications in hypertensive patient
}

\author{
Maria Leonarda DE ROSA ${ }^{1 *}$, Marta CERINI ${ }^{2}$ and Licia CERINI ${ }^{3}$ \\ ${ }^{1}$ Translational Medical Science Department, School of Medicine, University of Naples Federico II, Italy \\ ${ }^{2}$ Energy Department, Politecnico of Milan, Italy \\ ${ }^{3}$ Management and Technology Department, Bocconi University, Milan, Italy
}

\begin{abstract}
Objective: Cost-effectiveness evaluations or budget impact analysis represent a typical example of decision analysis problems. In this study, we aim to provide an introduction to these so called decision-theoretic networks. A formal introductory background is followed by an example of a cost-effectiveness evaluation of cardiovascular therapy.

Methods: Using a Markov CVD model, we compared 6 strategies for initiation of drug treatment-2 different blood pressure levels (165/95 and 145/90 mm Hg) and 4 different levels of absolute cardiovascular risk over 10 years (45\%,35\%, 25\%, and 15\%). We modeled a hypothetical cohort of all adults without cardiovascular diseases in Italy over 10 years.

Results: The incremental cost-effectiveness ratios for treating those with 10 -year absolute risk for cardiovascular diseases $>45 \%$, 35\%, 25\%, and 15\% were Euro 450, 1000, 2800, and 6000 ,respectively per quality-adjusted life-year (QALYs)gained, respectively. Strategies based on a target blood pressure level were both more expensive and less effective than treatment decisions based on the strategy that used absolute cardiovascular risk of $>15 \%$. Sensitivity analysis of cost of treatments, prevalence estimates of risk factors, and benefits expected from treatment did not change the ranking of the strategies. National inpatient hospital costs for hypertensive patient with complications were nearly Euro 2.8 billion in 2012.
\end{abstract}

Conclusions: This may suggest that quality of life is a more subjective and less tangible outcome for decision-makers than patients cured, strokes averted, or years of life saved. QALYs are the preferred outcome measures in a number of formal guidelines for conducting pharmacoeconomic analyses.

\section{Introduction}

The rapidly rising cost of drug therapy is a concern to healthcare providers throughout the world, particularly as new drugs frequently offer only marginal improvements over existing therapies but at substantially increased costs [1]. As a result, decision-makers at all levels (national, regional, hospital, primary care, or managed care) are faced with difficult choices about which drugs to make available to their patients or members. One means of controlling costs is the development of a formulary, resulting from explicit decision-making to include or exclude particular medications from the range of drugs available to be prescribed. Several decision-making criteria and methods for selecting drugs for formulary inclusion have been suggested, but the role of pharmacoeconomic information in this process remains somewhat unclear. The World Health Organization (WHO) describes broad criteria to be considered in making formulary decisions but does not attach relative importance to the individual criteria [2]. Decisions about drug selection are complex and are influenced not just by evidencebased criteria but also take into account the political, social, and ethical values of the community to which the decisions apply. A number of frameworks have been proposed to limit the influence of "emotional criteria" on decision-making and to assign relative weight to each criterion in terms of importance [3]. Another formal scoring system based on quality and cost factors has been used to assign priorities for purchasing drugs within a limited hospital drug budget [4]. "Quality" components in this model include individual patient benefit, mortality/ morbidity of the condition to be treated, evidence from literature, type of treatment (well-established or trial), and clinical comparison with other treatments. "Cost" components include comparison with other treatments, total cost per year, and cost per patient. The similarities in the key criteria examined (efficacy, safety, drug characteristics, pharmacokinetics, formulation, dosage regimen) suggest the possibility of a decision-making framework that could be broadly applicable across settings, both in developed and developing countries. An area less clearly articulated, however, is where and how cost information should be incorporated in decision-making. An increasing number of national or regional organizations now require pharmacoeconomic information about a drug to be included in submissions for reimbursement or listing [5]. Despite advances in treatment, cardiovascular disease is expected to be the leading cause of death and disability worldwide by 2020 [6]. Among risk factors for cardiovascular disease, hypertension is responsible for more deaths worldwide than any other, including cholesterol, tobacco, body mass index, and physical activity [7]. Hence, the social and economic demands of adequately treating hypertension are of considerable magnitude and complexity. The blood pressure level-approach based on a single cutoff point does not differentiate risk

Correspondence to: Maria Leonarda De Rosa, Translational Medical Science Department, School of Medicine, University of Naples Federico II, Italy, Tel/Fax: 0039-081-7145679; E-mail: mlderosa@unina.it

Key words: cost-benefit analysis, hypertension, prevention, cardiovascular diseases, quality of life

Received: August 24, 2017; Accepted: September 11, 2017; Published: September 14, 2017 
based on age, actual level of cholesterol, smoking, or the number of other risk factors present. Consequently, this method does not allow for precise assessment of a patient's true cardiovascular risk. The WHO CHOICE (World Health Organization Choosing Interventions that are Cost-Effective program) program showed that the strategy of treating those at high absolute risk in various regions of the world was cheaper and saved more lives than one based on target levels of individual risk factors. However, the model is limited by its use of regional rather than national epidemiology and cost estimates. These limitations are particularly important because the distribution of hypertension differs by geography and ethnicity, as much as does the extent of economic development in different countries [8,9]. Cost-effectiveness evaluations represent a typical example of such a problem, as they are performed for decision making regarding reimbursement of drugs or health care budget allocation, in presence and under the influence of random variables. Standard tools for pharmacoeconomic evaluations include regression techniques and decision-analytic models, e.g. decision trees, Markov state-transition models, and discrete event simulations. Decision trees are so far the most used tool to support decision making under uncertainty. The decision problem is represented by means of a tree-like structure combined with conditional probabilities and utilities (e.g. costs, quality of life multipliers) associated with the different decisions. Decision trees have a direct connection to expected utility theory, and are generally good for (deterministic) sensitivity analysis [10,11].

\section{Methods}

We conducted a cost-effectiveness analysis of blood pressure level- and absolute risk-based hypertension guidelines in persons without known cardiovascular disease or target-organ disease [12]. The strategies compared included: two different strategies based on blood pressure levels (initiation of treatment for those with blood pressure higher than $165 / 95$ or $145 / 90 \mathrm{~mm} \mathrm{Hg}$ and diabetes mellitus), four different strategies based on the absolute level of cardiovascular risk over ten years $(45 \%, 35 \%, 25 \%$ and $15 \%)$ according to the standard of care currently in practice. There have been no trials that compared the different hypertension guidelines. Even if these trial data did exist, they likely would not be for the length of time we chose to evaluate, and we therefore chose to model cardiovascular disease.

Seven thousands two hundred forty people, were selected from the database of outpatient clinic in primary health care in Federico II University Hospital of Naples, Italy. Patients were recruited for the study during their annual checkup at the outpatient clinic. All patients had a previously established diagnosis of essential hypertension (at least for 1 year). The possible causes of secondary hypertension were excluded (no clinical signs, normal results of serum urea, creatinine, and electrolytes, normal urinary excretion of catecholamines, and normal renal imaging studies, including radionuclide renogram and/or intravenous urography and/or ultrasound scan). All participants were white, were stratified into 20 groups representing each of the possible combinations of the 6 characteristics: sex, age, systolic blood pressure, smoking, diabetes mellitus, and cholesterol. Age was stratified into 5 deciles ( 34 to 44,45 to 55,56 to 66,67 to 77 and 78 to 88 years). There was further stratification by systolic blood pressure into 3 groups: $<140$ $\mathrm{mm} \mathrm{Hg}, 140$ to $160 \mathrm{~mm} \mathrm{Hg}$, and $>160 \mathrm{~mm} \mathrm{Hg}$. Single BP measurements were taken at baseline, and comorbidity and medication data were collected only at baseline. Further dichotomization was done for smoking and diabetes mellitus. Finally, the stratifications were divided according to 3 levels of total cholesterol: $<190 \mathrm{mg} / \mathrm{dL}, 190$ to $270 \mathrm{mg} /$ $\mathrm{dL}$, and $>270 \mathrm{mg} / \mathrm{dL}$. The distributions of cholesterol and diabetes mellitus were stratified with mean values according to sex, because few data exist to determine their relationship to the other risk factors.
A Markov model was used to assess the cost-effectiveness of the six alternative guidelines for hypertension in an hypothetical cohort of healthy individuals [13]. The population was stratified by gender, age, systolic blood pressure, smoking, diabetes mellitus and cholesterol. The time horizon of the model was ten years and the cycle length was one year. The population started the simulation free of cardiovascular disease (Figure 1). Every year individuals could remain disease free, die of non- cardiovascular disease causes, or develop fatal or nonfatal cardiovascular disease. At the end of each year, the population was redistributed to one of several health states, depending on the events of the previous year. Such health states were disease free (no cardiovascular disease event or death of other causes), post-arrest, post-MI without coronary artery bypass grafting (CABG), post-MI with $C A B G$, post-angina without $C A B G$, post-angina with $C A B G$, post-cerebrovascular accident, and death (both non- cardiovascular disease -related and cardiovascular disease -related death). Table 1 shows assessed outcomes.

In the economic analysis both future costs and benefits were discounted at $3 \%$ per year, consistent with current guidelines. Effects were measured in years of life and quality-adjusted life-years (QALYs) gained. Quality-adjusted life-years were the most appropriate benefit measure because they capture the impact of the intervention on the two most important dimensions of health for patients with hypertension (i.e. quality of life and survival). Quality-adjusted life-years are comparable with the benefits of other health care interventions [14].

\section{Sensitivity analysis}

Univariate sensitivity analyses were carried out to assess the robustness of cost-utility estimates to variations in the model inputs, such as -costs of hypertension treatment:- cost per hospital diagnosis;proportion of patients undergoing CABG or angiography;-proportion of the population receiving anti-hypertensive treatment;-prevalence of hypercholesterolemia ;-relative risk reduction from treatment and discount rate. A probabilistic multivariate sensitivity analysis was also performed, using Monte Carlo simulation, in which the previously mentioned inputs were varied simultaneously. Traditionally, with a decision tree (or Markov state-transition model) the most common way of performing probabilistic sensitivity analysis is by means of simulation procedures, such as Monte Carlo or Markov Chain Monte Carlo simulation. With these procedures, the model is iteratively analyzed. At each step, the input parameters are sampled from a suitable probability distribution, reflecting the likelihood of each input value. As a result, a distribution of the outcomes or expected utility is obtained, reflecting the underlying uncertainty, and allowing its quantification [15] (Figure 2).

\section{Results}

The proportion of eligible patients receiving anti-hypertensive treatment was $44 \%$ (range: 32 to 56 ). The reduction in systolic blood pressure with drug treatment was $10 \mathrm{mmHg}$. The cardiovascular disease risk reduction with drug treatment was $14 \%$ (range: 14 to 25 ), while the stroke risk reduction was $40 \%$ (range: 10 to 50). Table 2 shows, over a 10-year timeframe, the expected quality-adjusted lifeyears (in millions) in a population of 7240 individuals. In Figure 3 QALYs is presented by discrete second order probability distributions. When the nodes Probability Early Switch and Uncertainty Monetary value QALYs are instantiated at a certain value. Since the likelihood of the instantiated values of Probability Early Switch and Uncertainty 


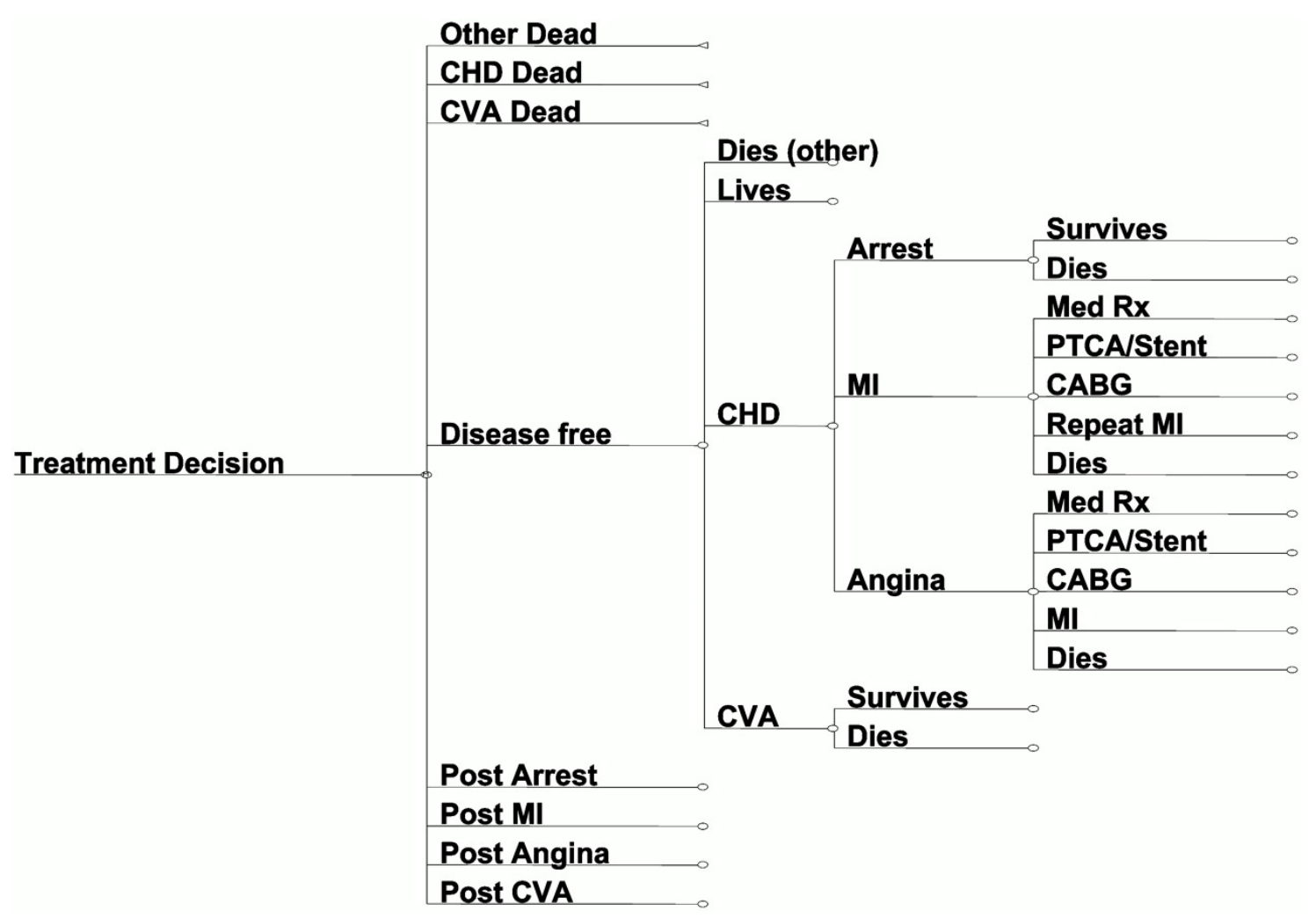

CHD: coronary heart disease; CVA: cerebrovascular accident; Med Rx treatment; MI: myocardial infarction.

Figure 1. Schematic of CVD model. The full model represented by this simplified version has 62 possible clinical pathways. All members of the cohort begin free of CVD disease. In the initial year, each member of the cohort has a certain probability based on treatment status risk factors of moving along 1 of 4 pathways: remaining disease free, dying of non-CVD causes, or developing fatal or nonfatal coronary heart disease or stroke. In the subsequent years, those with prior CVD follow a separate pathway with adjusted risks for recurrence of CVD or death based on age and prior event. All risks are updated annually based on age. Costs are captured along the way for treatment of hypertension and CVD events.

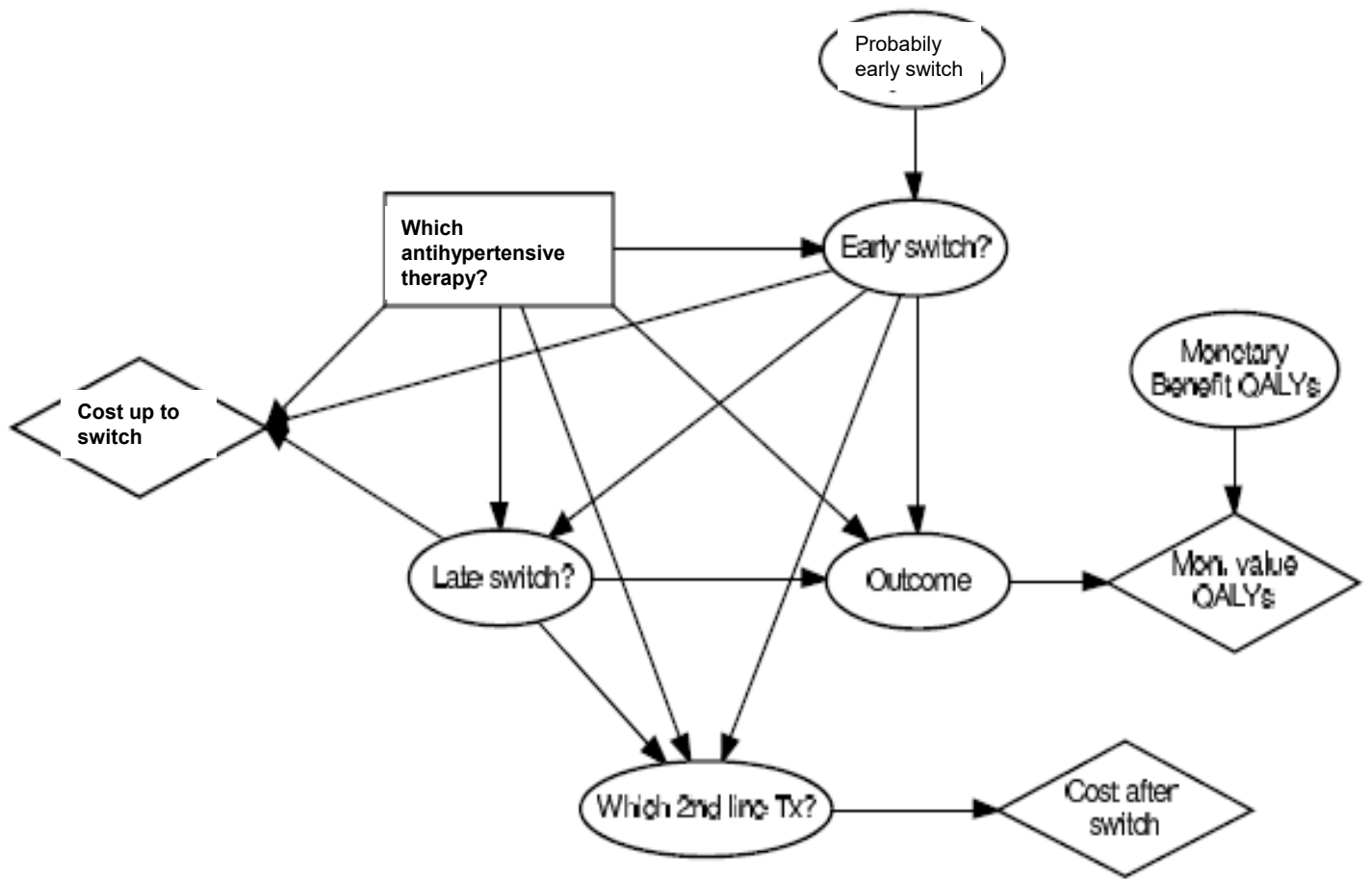

Figure 2. Influence diagram of antihypertensive treatment. 
Table 1. The utility adjustments used to calculate quality-adjusted life-years (QALYs) were also derived from the literature.

\begin{tabular}{|l|}
\hline Outcomes assessed \\
\hline -hypertension and smoking prevalence \\
\hline-10 -year CVD risk \\
\hline-10 -year coronary heart disease (CHD) risk \\
\hline-10 -year stroke risk \\
\hline -non-CVD mortality \\
\hline -the proportion of eligible people receiving anti-hypertensive treatment \\
\hline -treatment effects in terms of BP reduction \\
\hline -CHD reduction and stroke reduction \\
\hline
\end{tabular}

Table 2. Estimated benefits used in the economic analysis. Over a 10-year timeframe, the expected QALYs (in millions) in a cohort of 7240 individuals.

\begin{tabular}{|c|c|c|c|c|c|}
\hline & $\begin{array}{c}\text { Cost, } \\
\text { €Millions }\end{array}$ & $\begin{array}{c}\text { Incremental } \\
\text { Cost, }^{\dagger} \\
\text { €Millions }\end{array}$ & $\begin{array}{c}\text { Effect, } \\
\text { QALYs } \\
\text { (Millions) }\end{array}$ & $\begin{array}{c}\text { Incremental } \\
\text { Effect, }^{\dagger} \\
\text { QALYs }\end{array}$ & $\begin{array}{c}\text { Incremental } \\
\text { C/E Ratio } \\
\text { €/QALY }\end{array}$ \\
\hline No treatment & 3460 & $\ldots$ & 44.694 & $\ldots$ & $\ldots$ \\
\hline $\begin{array}{l}\text { Absolute risk } \\
\text { CVD }>40 \%\end{array}$ & 3464 & 4 & 44.700 & 6.000 & 700 \\
\hline $\begin{array}{l}\text { Absolute risk } \\
\text { CVD }>30 \%\end{array}$ & 3491 & 27 & 44.721 & 21.000 & 1300 \\
\hline $\begin{array}{l}\text { Absolute risk } \\
\text { CVD }>20 \%\end{array}$ & 3671 & 180 & 44763 & 42.000 & 4300 \\
\hline $\begin{array}{l}\text { Absolute risk } \\
\text { CVD }>15 \%\end{array}$ & 3746 & 85 & 44.781 & 18.000 & 6.000 \\
\hline $\begin{array}{l}\text { 2000 Italian } \\
\text { guidelines } \\
\text { (target level } \\
160 / 95 \mathrm{~mm} \mathrm{Hg} \text { ) }\end{array}$ & 3900 & 154 & 44.760 & -21000 & Dominated $^{\ddagger}$ \\
\hline $\begin{array}{c}\text { Current } \\
\text { guidelines } \\
\text { (target level } \\
145 / 90 \mathrm{~mm} \mathrm{Hg} \text { ) }\end{array}$ & 4200 & $300^{\S}$ & 44.279 & -2000 & Dominated \\
\hline
\end{tabular}

$\dagger$ Each strategy's cost and effect are compared with the preceding less costly one. \# dominated strategy is one that is both more expensive and less effective than the preceding strategy to which it is compared.

$\S$ Compared with absolute risk CVD $>15 \%$, because the 2000 guidelines are dominated by the absolute risk CVD $>15 \%$ strategy.

Monetary value QALYs is defined by their marginal distributions the likelihood of expected utility is obtained free of charge. For example, $\operatorname{Pr}$ (Probability Early switch $=0.05$ ) equals $6.63 \%$ and $\operatorname{Pr}$ (Uncertainty Monetary value QALYs = e250,000) equals 6.25\% for antihypertensive therapy. The corresponding expected utility is e151,752 with a likelihood of $0.40 \%$. Over a 10 -year timeframe, the expected costs (in millions) in a population of 7240 individuals were showed in table 3.

\section{Synthesis of costs and benefits}

Incremental cost-effectiveness ratios and cost-utility ratios were calculated to combine the costs and benefits of each strategy with the next less effective option. The two strategies based on target BP levels were dominated as they were both less effective and more expensive than absolute risk options. Again, the two strategies based on target blood pressure levels were dominated. The sensitivity analysis showed that changes in model inputs did not alter the ranking of the strategies. Only when the annual cost of hypertension treatment fell below the threshold point of $€ 30$ did the strategy with absolute CVD risk of > $40 \%$ become less expensive and more effective than the no-intervention strategy. In this high-risk population, the number needed to treat to prevent 1 event was low enough that below $€ 30$ per year of treatment, the reduction in costs from preventing events was greater than the cost of treating those who would not have an event over the 10 years. Above $€ 30$ per year of antihypertensive therapy, the ranking of the strategies was the same as in the base case, with no treatment having the lowest costs and benefits and the 4 absolute risk strategies each with successive incremental cost-effectiveness ratiosThe multivariate sensitivity analysis showed that the base-case results were quite robust to variations in model assumptions. National inpatient hospital costs for hypertensive patient with complications were nearly Euro 2.8 billion in 2012. The risk of hospitalization from cardiovascular disease is two to four times higher for women with diabetes and hypertension compared to women without diabetes.

\section{Discussion}

In this paper, we presented an introduction to decision-theoretic networks, with specific applications to cost- effectiveness evaluations. While this tool has been widely used in other research fields, it has been so far neglected by the pharmacoeconomics community. The clinical experts play a crucial role in the definition of the network structure, and joining statisticians or economists in drawing a graph is surely easier (and funnier) than writing mathematical equations. We reckon that statistical, economic and clinical research is to be focused on that topic in the next future. A decision network (an equivalently, an influence diagram) is a belief network that is augmented to represent the alternative actions available to a decision maker (with decision nodes) and his preference for alternative states (with a value node). In terms of the construction of network, we would like to be able to construct the value node from sentences in the our database as well as incorporate decision nodes in the construction process. A value function is a mapping from the various possible outcomes or states resulting in particular decision problem to a single real number. In a decision network, there is a single value node. A value function for medical treatment might look something like the following, where Patients value $(v, p)$ stands for the relation "Patient $p$ 's value is $v$ ". The value $v$ is the value variable in this predicate. Direct specification of such a table of possible decision a situation is unwieldly and furthermore is subject to exactly the type of inflexibility a lack of context sensitivity that we encounter with fixed probabilistic model. We therefore construct these structures based on the set of deterministic dependencies, facts and alternative outcomes in the database.

Another interesting feature of decision-theoretic networks is that, since they represent a model for the entire joint probability distribution of a set of variables, there is no need for the definition of "dependent" (response) and "independent" (covariates) variables. Each node is evaluated in terms of the dependence relations with the others, overcoming the limitation of some regression models, where a given form of causality is given as an assumption, without the possibility of learning over it [16]. Therefore, decision-theoretic networks distinguish themselves from standard regression models as they try to manifest not only the correlation between the covariates and the response variable, but also the correlation between the different covariates. This is useful both when the researcher tries to capture the effects of indirect influences, and when some important covariate is missing from the dataset. While we strongly advocate the use of graphical models, we

Table 3. Cost results. Over a 10-year timeframe, the expected costs (in millions) in a population of 7240 individuals were.

$€ 460$ with no intervention,

$€ 450$ with the strategy of drug intervention for absolute CVD risk of $>45 \%$,

$€ 1000$ with absolute CVD risk of $>35 \%$,

$€ 2800$ with absolute CVD risk of $>25 \%$,

$€ 6000$ with absolute CVD risk of $>15 \%$,

$€ 6100$ with the strategy of drug intervention for BP level higher than $165 / 95 \mathrm{mmHg}$, and $€ 6,735$ with a target BP level of $145 / 90 \mathrm{mmHg}$ 


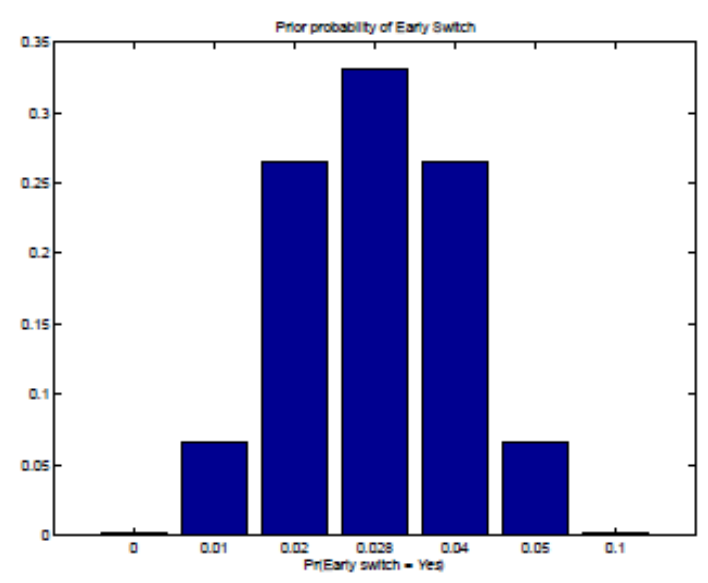

(a)

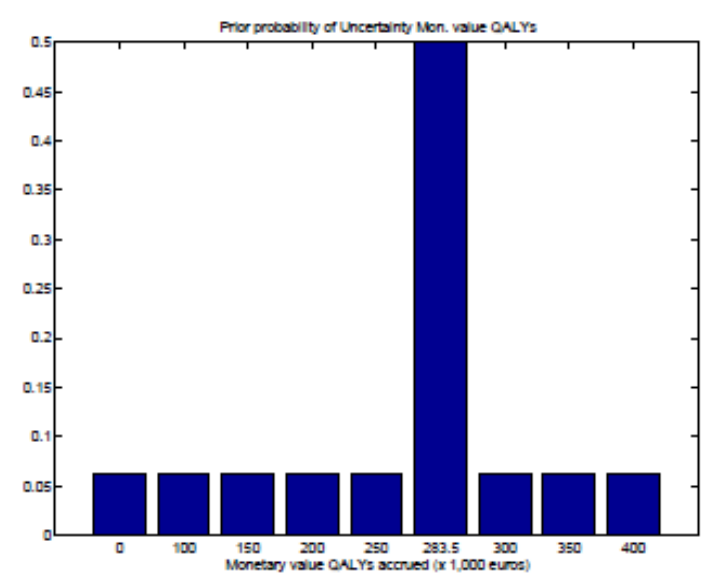

(b)

Figure 3. Prior probability distribution for probability early switch and uncertainty mon value QALYs. Graphs are presented for antihypertensive therapy.

acknowledge some limitations of decision-theoretic networks. Unlike decision trees assumes symmetry (i.e. every scenario consists of the same sequence of variables). Moreover, complex problems may require a large number of probability assessments [17]. On the one hand, it is often the case that variables of interests (such as rates of occurrence of a given disease, or costs associated with this disease) are naturally defined over a continuous scale. However, it is possible to approximate this reality, making these variables discrete (possibly using a large grid of plausible values). On the basis of the results of our population-based simulation model, we conclude that for the current Italian population, hypertension guidelines based on an absolute risk for cardiovascular disease are both more effective at saving lives and less costly than those based predominantly on a blood pressure level. This approach is even more attractive if the cost of treatment could be reduced. We modeled the cost of hypertension treatment based on the current mix of medications used in Italy. If the use of thiazides replaces the more expensive calcium channel blockers or ACE inhibitors, then the cost-effectiveness ratios for primary prevention will be further reduced, and the absolute risk strategies will be even more attractive, if not economically dominant, compared with no treatment. Furthermore, the conventional blood pressure-based guidelines remained more costly and less effective with each univariate and multivariate sensitivity analysis. We recognize that our model is based on limited cost data, which is a significant problem throughout the developing world. However, given that we applied the same costs of treatment to all the intervention strategies, the results seen in the sensitivity analysis still showed that the blood pressure level guidelines were always dominated throughout the assessed ranges of costs. Certainly, the costs of interventions could be much lower if generic medications were used instead of the current pattern of use of both patented and generic drugs in Italy. Thus, our estimates are likely conservative. However, under this criterion, the absolute risk of $15 \%$ strategy would also be acceptable if a greater proportion of generic medications, such as hydrochlorothiazide, were used. Antihypertensive medication may be expected to have two opposing effects on quality of life: improvements through the reduced incidence of cardiovascular events and reductions through the impact of treatment-related adverse effects. The latter could potentially be important in assessing the balance between benefits and harms, particularly for low-risk individuals. Differences in adverse effects between the drugs could also have an influence on their relative cost effectiveness. A Medline search was done to identify utility estimates that could be used to reflect the latter for the included drug classes. Some studies were identified that estimated the incidence of drug-related adverse events and quality of life $[18,19]$. However, none of these included data in a form suitable for estimation of utilities. Most published cost-effectiveness studies have assumed zero, or minimal (0.01), loss of quality of life due to treatment-related side effects according to Harvard CEA Registry264 [20]. Where these have compared different antihypertensive medications, they have generally assumed equal utility loss from adverse effects of treatment [21]. Few studies have directly measured treatment utilities from patients. The economic analysis of the SCOPE trial included direct assessment of utility using the EuroQoL health status measurement instrument [22]. This estimated a mean change in utility of minus 0.03 for the candesartan group and minus 0.05 for the mixed hypertensive treatment control group over a mean followup of 3.7 years. However, it is not possible to separate out the impact of treatment side effects, or to attribute utility losses to individual drugs. Another cost-effectiveness study estimated utilities from 148 hypertensive patients using the standard gamble technique. They found a net loss in utility of 0.027 , but did not report any difference by drug [23]. The present findings are consistent with the WHO CHOICE by Murray, et al. [6]: the absolute risk approach also had lower costeffectiveness ratios than the target-level approach. The present study extends their findings in distinct ways. First, we looked at a specific country with more detailed epidemiological and cost data. This allows an individual country like Italy to shift resources based on its specific health needs and budgetary limitations. It allows a direct comparison of the two possible approaches under consideration and evaluation of what a change from its current approach to a new one might entail. Second, our model included inpatient costs of cardiovascular events. Given that these events are responsible for a significant proportion of costs related to a blood pressure and cardiovascular disease prevention program, our costs more likely reflect actual costs associated with the intervention. Additionally, a recent study in a busy primary care setting in the United Kingdom found that use of a chart based on absolute risk calculations was well-liked, easy to use, and resulted in a clinically significant reduction in blood pressure compared with usual care [24]. One limitation is that the absolute risk approach uses the Framingham function, which is based on a predominantly US white population. However, it has been shown to be equally predictive among white and 
black men and women in the United States [25]. Furthermore, the case-control INTERHEART study confirmed that similar risk factors accounted for the majority of myocardial infarction regardless of region [26]. Further generalization of our results to other developing countries should take into account the fact that the risk score may overestimate or underestimate disease, which would increase or decrease the incremental cost-effectiveness ratios, respectively.

\section{Conclusions}

We stated that our results were comparable with the findings published in a study from the WHO CHOICE program. In terms of the issue of the generalizability of the study results to other developing countries, we noted that risk scores might overestimate or underestimate disease, thus caution is required when extrapolating the results of the study to other settings. Several sensitivity analyses might be performed, which further enhance the external validity of the study. A potential limitation of the analysis was that the estimation of risk factors was based on a predominantly Italian white population. Hypertension guidelines based on an absolute risk for cardiovascular disease could be both more effectiveness and less expensive than guidelines based mainly on blood pressure level. Reductions in the cost of antihypertensive drugs could even make this guideline costsaving in comparison with no intervention. The study results support the use of hypertension guidelines based on the assessment of absolute cardiovascular risk among healthy individuals.

\section{References}

1. Kessler DA, Rose JL, Temple RJ, Schapiro R, Griffin JP (1994) Therapeutic-class wars--drug promotion in a competitive marketplace. $N$ Engl J Med 331: 1350-1353. [Crossref]

2. Holloway K, Green T (2003) Drug and therapeutics committees: A practical guide. Geneva, Switzerland: World Health Organization.

3. Janknegt R, Steenhoek A (1997) The system of objectified judgement analysis (SOJA). A tool in rational drug selection for formulary inclusion. Drugs 53: 550-562. [Crossref]

4. Bochner F, Martin ED, Burgess NG, Somogyi AA, Misan GM (1994) Controversies in treatment: How can hospitals ration drugs? Drug rationing in a teaching hospital: A method to assign priorities. BMJ 308: 901-905.

5. Drummond M, Brown R, Fendrick AM, Fullerton P, Neumann P, et al. (2003) Use of pharmacoeconomics information-Report of the ISPOR task force on use of pharmacoeconomic/health economic information in health-care decision making. Value Health 6: 407-416. [Crossref]

6. Murray CJ, Lopez AD (1997) Alternative projections of mortality and disability by cause 1990-2020: Global burden of disease study. Lancet 349: 1498-1504. [Crossref]

7. Ezzati M, Lopez AD, Rodgers A, Vander Hoorn S, Murray CJ, Comparative risk assessment collaborating group (2002) Selected major risk factors and global and regional burden of disease. Lancet 360: 1347-1360. [Crossref]

8. Murray CJ, Lauer JA, Hutubessy RC, Niessen L, Tomijima N, Rodgers A, et al. (2003) Effectiveness and costs of interventions to lower systolic blood pressure and cholesterol: a global and regional analysis on reduction of cardiovascular-disease risk. Lancet 361: 717-725. [Crossref]
9. Yusuf S, Reddy S, Ounpuu S, Anand S (2001) Global burden of cardiovascular diseases, part I: general considerations, the epidemiologic transition, risk factors, and impact of urbanization. Circulation 104: 2746-2753. [Crossref]

10. Raiffa H (1968) Decision analysis. Addison-Wesley, Reading, MA, USA.

11. Fenwick E, O’Brien BJ, Briggs A (2004) Cost-effectiveness acceptability curves facts, fallacies and frequently asked questions. Health Econ 13: 269-274. [Crossref]

12. Mancia G, Fagard R, Narkiewicz K, Redon J, Zanchetti A, et al. (2013) 2013 ESH/ ESC guidelines for the management of arterial hypertension: The Task Force for the management of arterial hypertension of the European Society of Hypertension (ESH) and of the European Society of Cardiology (ESC). Eur Heart J 34: 2159-2219. [Crossref]

13. Sonnenberg FA, Beck JR (1993) Markov models in medical decision making: a practical guide. Med Decis Making 13: 322-338. [Crossref]

14. Drummond M, Sculpher M (2005) Common methodological flaws in economic evaluations. Med Care 43: 5-14. [Crossref]

15. Spiegelhalter DJ, Abrams KR, Myles JP (2004) Bayesian Approaches to Clinical Trials and Health-Care Evaluation. Chichester (UK): John Wiley \& Sons.

16. Fenwick E, O'Brien BJ, Briggs A (2004) Cost-effectiveness acceptability curves facts, fallacies and frequently asked questions. Health Econ 13: 269-274. [Crossref]

17. Baker S, Priest P, Jackson R (2000) Using thresholds based on risk of cardiovascular disease to target treatment for hypertension: modelling events averted and number treated. BMJ 320: 680-685. [Crossref]

18. Hollenberg NK, Williams GH, Anderson R, Akhras KS, Bittman RM, Krause SL (2003) Symptoms and the distress they cause: comparison of an aldosterone antagonis and a calcium channel blocking agent in patients with systolic hypertension. Arch Intern Med 163: 1543-1548. [Crossref]

19. Van Bortel LM, Bulpitt CJ, Fici F (2005) Quality of life and antihypertensive effect with nebivolol and losartan. Am J Hypertens 18: 1060-1066. [Crossref]

20. National Clinical Guideline Centre (2011) Cost-effectiveness analysis pharmacological treatment (updated 2011). Hypertension 421-445.

21. Jönsson B, Carides GW, Burke TA, Dasbach EJ, Lindholm LH, et al. (2005) Cost effectiveness of losartan in patients with hypertension and LVH: an economic evaluation for Sweden of the LIFE trial. J Hypertens 23: 1425-1431. [Crossref]

22. Degl'innocenti A, Elmfeldt D, Hofman A, Lithell H, Olofsson B, Skoog I, et al. (2004) Health-related quality of life during treatment of elderly patients with hypertension: results from the study on cognition and prognosis in the elderly (SCOPE). J Hum Hypertens 18: 239-245. [Crossref]

23. Montgomery AA, Fahey T, Ben-Shlomo Y, Harding J (2003) The influence of absolute cardiovascular risk, patient utilities, and costs on the decision to treat hypertension: a Markov decision analysis. J Hypertens 21: 1753-1759. [Crossref]

24. Montgomery AA, Fahey T, Peters TJ, MacIntosh C, Sharp DJ (2000) Evaluation of computer based clinical decision support system and risk chart for management of hypertension in primary care: randomized controlled trial. BMJ 320: 686-690. [Crossref]

25. D'Agostino RB Sr, Grundy S, Sullivan LM, Wilson P, CHD Risk Prediction Group (2001) Validation of the Framingham coronary heart disease prediction scores: results of a multiple ethnic groups investigation. JAMA 286: 180-187. [Crossref]

26. Yusuf S, Hawken S, Ounpuu S, Dans T, Avezum A, et al. (2004) Interheart Study Investigators. Effect of potentially modifiable risk factors associated with myocardial infarction in 52 countries (the INTERHEART study): case-control study. Lancet 364 937-952. [Crossref]

Copyright: C2017 De Rosa ML. This is an open-access article distributed under the terms of the Creative Commons Attribution License, which permits unrestricted use, distribution, and reproduction in any medium, provided the original author and source are credited. 